\title{
RATIONAL FUNCTIONS WITH PRESCRIBED CRITICAL POINTS
}

\author{
I. SCHERBAK \\ School of Mathematical Sciences, Tel Aviv University, \\ Ramat Aviv, Tel Aviv 69978, Israel \\ e-mail: scherbak@post.tau.ac.il
}

\begin{abstract}
A rational function is the ratio of two complex polynomials in one variable without common roots. Its degree is the maximum of the degrees of the numerator and the denominator. Rational functions belong to the same class if one turns into the other by the postcomposition with a linear-fractional transformation. We give an explicit formula for the number of classes having a given degree $d$ and given multiplicities $m_{1}, \ldots, m_{n}$ of given $n$ critical points, for generic positions of the critical points. This number is the multiplicity of the irreducible $s l_{2}$ representation with highest weight $2 d-2-m_{1}-\cdots-m_{n}$ in the tensor product of the irreducible $s l_{2}$ representations with highest weights $m_{1}, \ldots, m_{n}$.

The classes are labeled by the orbits of critical points of a remarkable symmetric function which first appeared in the XIX century in studies of Fuchsian differential equations, and then in the XX century in the theory of KZ equations.
\end{abstract}

\section{INTRODUCTION}

A rational function is the ratio of two complex polynomials in one variable without common roots. Its degree is the maximum of the degrees of the numerator and the denominator. Any rational function defines a branched covering of the Riemann sphere, $\mathbb{C P}^{1}=\mathbb{C} \cup \infty$, and the degree is the cardinality of the preimage of any regular value.

Let $R(x)=g(x) / f(x)$, where $g(x)$ and $f(x)$ are polynomials without common roots, be a rational function of degree $d$. We have $R^{\prime}(x)=W(x) / f^{2}(x)$, where

$$
W(x)=W[g, f](x)=g^{\prime}(x) f(x)-g(x) f^{\prime}(x)
$$

is the Wronskian of polynomials $g$ and $f$.

The rational function $R(x)$ has a critical point of multiplicity $m \geq 1$ at $z \in \mathbb{C}$, if $z$ is a root of the Wronskian $W(x)$ of multiplicity $m$. Let $m_{1}, \ldots, m_{n}$ be positive integers and $z_{1}, \ldots, z_{n}$ pairwise distinct complex numbers. Write $\mathbf{m}=\left(m_{1}, \ldots, m_{n}\right)$, $M=m_{1}+\cdots+m_{n}$ and $\mathbf{z}=\left(z_{1}, \ldots, z_{n}\right)$. If $R(x)$ has degree $d>1$ and if all its finite critical points are $z_{1}, \ldots, z_{n}$ of multiplicities $m_{1}, \ldots, m_{n}$ respectively, then we say that $R(x)$ has the type $(d, n ; \mathbf{m} ; \mathbf{z})$. According to the Riemann-Hurwitz formula, numbers $d$ 
and $M$ should satisfy $2 d-2 \geq M$. The difference $m_{\infty}=2 d-2-M$ is the multiplicity of $R(x)$ at infinity.

If $R(x)$ has the type $(d, n ; \mathbf{m} ; \mathbf{z})$, then the rational function given by the postcomposition with a linear-fractional transformation,

$$
\frac{a R(x)+b}{c R(x)+d}, \quad a d-b c \neq 0,
$$

has clearly the same type. A rational function considered up to postcompositions with linear-fractional transformations will be called a class of rational functions.

We address the following question.

Given $d, n, \mathbf{m}, \mathbf{z}$, how many classes have the type $(d, n ; \mathbf{m} ; \mathbf{z})$ ?

The question is easy for $n=1$. If $z \in \mathbb{C}$ is the single critical point, then the number of classes is 1 if the multiplicity of $z$ is $d-1$ (this is the class containing $(x-z)^{d}$ ), and 0 otherwise.

If $n \geq 2$, we answer the question for generic $\mathbf{z}$. The words " $\mathbf{z}$ is generic" mean that $\mathbf{z}$ does not belong to a suitable proper algebraic set in $\mathbb{C}^{n}$.

Theorem. Let $n \geq 2$ and $d, m_{1}, \ldots, m_{n}$ be positive integers. For generic $\mathbf{z}$, the number $\sharp(d, n ; \mathbf{m})$ of classes of rational functions of the type $(d, n ; \mathbf{m} ; \mathbf{z})$ is

$$
\sharp(d, n ; \mathbf{m})=\sum_{q=1}^{n}(-1)^{n-q} \sum_{1 \leq i_{1}<\cdots<i_{q} \leq n}\left(\begin{array}{c}
m_{i_{1}}+\cdots+m_{i_{q}}+q-d-1 \\
n-2
\end{array}\right),
$$

and any nonempty class can be represented by the ratio of polynomials without multiple roots.

As usually we set $\left(\begin{array}{l}a \\ b\end{array}\right)=0$ for $a<b$.

\section{Remarks:}

- If $m_{j}>d-1$ for some $j$, or if $M>2 d-2$, or if $M<d-1$, then the right hand side of (11) vanishes. In fact, in this case for any $\mathbf{z}$ there are no rational functions of the type $(d, n ; \mathbf{m} ; \mathbf{z})$. Indeed, any of the inequalities is impossible for a rational map of degree $d$. This was known, see Ch. 6 of [GH] or Lemma 1.1 of [G].

- If $M=d-1$ and $m_{j} \leq d-1$ for $j=1, \ldots, n$, then the right hand side of (11) gives 1. Again, for any $\mathbf{z}$ the number of classes is 1. Indeed, if $R(x)=g(x) / f(x)$ belongs to a corresponding class, and if $g$ has degree $d$, then the degree of $f$ is clearly zero, i.e. this class contains the polynomial

$$
\int\left(x-z_{1}\right)^{m_{1}} \ldots\left(x-z_{n}\right)^{m_{n}} d x
$$


- The case when the number of critical points is the maximal possible, i.e. $n=$ $2 d-2$ and $m_{1}=\cdots=m_{n}=1$, was studied by L. Goldberg in G. Denote $\mathbf{1}=(1, \ldots, 1)$. Theorem 1.3 of [G] says that $\sharp(d, 2 d-2 ; \mathbf{1})=\frac{1}{d}\left(\begin{array}{c}2 d-2 \\ d-1\end{array}\right)$, which is the $d$-th Catalan number.

- The condition " $\mathbf{z}$ is generic" is essential. For some of $\mathbf{z}$, the number of classes may decrease. Actually, the inequality (3) and the left-hand inequality of (6) hold for any $\mathbf{z}$ whereas the right-hand inequality of (6) may be broken for some of $\mathbf{z}$. An example is given in Theorem 1.4 of [G], cf. Sec. 12 of [RV].

In view of the first and second remarks, we assume

$$
d-1<M \leq 2 d-2, \quad 1 \leq m_{j} \leq d-1, \quad j=1, \ldots, n .
$$

The proof of the theorem consists of the following three steps.

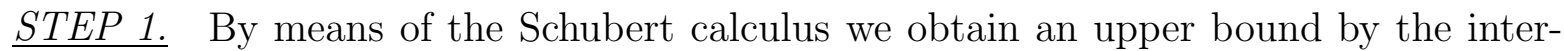
section number of special Schubert classes $\sigma_{m_{1}}, \ldots, \sigma_{m_{n}}, \sigma_{2 d-2-M}$ :

$$
\sharp(d, n ; \mathbf{m}) \leq \sigma_{m_{1}} \cdot \ldots \cdot \sigma_{m_{n}} \cdot \sigma_{2 d-2-M} .
$$

This step is done in Sec. 2.

STEP 2. Denote $L_{m_{j}}$ the irreducible $s l_{2}$-module with highest weight $m_{j}$, and $\operatorname{Sing}_{k} L$ the subspace of singular vectors of weight $M-2 k$ in the tensor product $L=L_{m_{1}} \otimes \cdots \otimes$ $L_{m_{n}}$. Well-known relations of the Schubert calculus to the representation theory $([\mathbb{E}])$ imply

$$
\sigma_{m_{1}} \cdot \ldots \cdot \sigma_{m_{n}} \cdot \sigma_{2 d-2-M}=\operatorname{dim} \operatorname{Sing}_{M+1-d} L .
$$

The number $\operatorname{dim} \operatorname{Sing}_{k} L$ was calculated in Theorem 5 of [SV]:

$$
\operatorname{dim} \operatorname{Sing}_{k} L=\sum_{q=0}^{n}(-1)^{q} \sum_{1 \leq i_{1}<\cdots<i_{q} \leq n}\left(\begin{array}{c}
k+n-2-m_{i_{1}}-\cdots-m_{i_{q}}-q \\
n-2
\end{array}\right) .
$$

In order to replace the inequality (3) with the equality one needs a non-trivial transversality statement which we did not find in the algebraic geometry literature $\mathrm{f}$.

We extract a lower bound from the results of N. Reshetikhin and A. Varchenko on the $s l_{2}$ Knizhnik-Zamolodchikov (KZ) equation, [RV]. The key observation is that rational functions and asymptotic solutions to the $s l_{2} \mathrm{KZ}$ equations turn to be linked by a remarkable symmetric master function whose critical point orbits determine both classes of rational functions and asymptotic solutions to the $s l_{2} \mathrm{KZ}$ equations. This is the

\footnotetext{
${ }^{1}$ As the author learned from the referee report, after the first version of this paper was posted on arXiv and submitted to this journal, a preprint of B. Osserman has appeared. The preprint contains an algebraic geometry proof of the equality $\sharp(d, n ; \mathbf{m})=\sigma_{m_{1}} \cdot \ldots \cdot \sigma_{m_{n}} \cdot \sigma_{2 d-2-M}$ based on the results of D. Eisenbud and J. Harris, [EH].
} 
very function studied by Heine and Stieltjes in connection with second order Fuchsian differential equations having a polynomial solution of a prescribed degree.

$\underline{S T E P ~ 3 .}$ Denote $\{\mathrm{NCO}\}$ the number of orbits of critical points of the master function given by (10). We get

$$
\sharp(d, n ; \mathbf{m}) \geq\{\mathrm{NCO}\} \geq \operatorname{dim} \operatorname{Sing}_{M+1-d} L .
$$

The left-hand inequality follows from the classical result of Heine and Stieltjes, Ch. 6.8 of [Sz]. We describe the connection between rational functions and Fuchsian equations and prove this inequality in Sec. 3. The right-hand inequality is proved in Theorem 9.9 of [RV] (cf. Theorem 8 of [SV]). The appearance of the master function in the theory of $\mathrm{KZ}$ equations is explained in Sec. 4 , following [RV].

Juxtaposing relations (3) $-($ (6) we arrive at the result.

Sec. 5 contains comments. In particular, connections of the Schubert calculus with Fuchsian equations having only polynomial solutions (Sec. 5.4) and with $s l_{p} \mathrm{KZ}$ equations (Sec. 5.5) are discussed.

Acknowledgments. The author is grateful to A. Eremenko, A. Gabrielov, B. Shapiro, M. Sodin, F. Sottile, A. Vainshtein, A. Varchenko, V. Zakalyukin, and the referee for useful comments and discussions.

\section{An upper bound: SCHubert Classes}

2.1. Classes of rational functions and generic planes in the Grassmannian. Denote $G_{2}$ (Poly) the Grassmannian of two-dimensional planes in the vector space of complex polynomials in one variable. Any plane $V \in G_{2}$ (Poly) has a basis of polynomials of distinct degrees. Define the degree of $V$ as the maximal degree of polynomials in $V$, and the order of $V$ as the minimal degree of non-zero polynomials in $V$. Say that $V \in G_{2}$ (Poly) is a generic plane, if for any $z \in \mathbb{C}$ there exists a polynomial $p(x) \in V$ such that $p(z) \neq 0$.

Proposition 1. There is a one-to-one correspondence between the generic planes and the classes of rational functions of the same degree.

Proof: $\quad V \in G_{2}$ (Poly) is generic if and only if the polynomials of any basis of $V$ do not have common roots. Any rational function $R(x)=g(x) / f(x)$ clearly defines a generic plane $V_{R}=\operatorname{Span}\{g(x), f(x)\}$ of the same degree. Conversely, any basis $\{g(x), f(x)\}$ of a generic plane $V$ of degree $d$ defines a rational function $R_{V}(x)=g(x) / f(x)$ of degree $d$. Linear-fractional transformations of the rational function correspond to changes of the basis. 
2.2. The Wronski map. Define the Wronskian of $V \in G_{2}$ (Poly) as a monic polynomial which is proportional to the Wronskian of some (and hence, any) basis of $V$. The question posed in the Introduction can be reformulated as follows:

Given monic polynomial $W(x)$ and given positive integer $d$, how many generic planes of degree d have the Wronskian $W(x)$ ?

It is enough to consider the $(d+1)$-dimensional vector space Poly ${ }_{d}$ of polynomials of degree $\leq d$ and the Grassmannian $G_{2}\left(\right.$ Poly $\left._{d}\right)$ of two-dimensional subspaces in Poly ${ }_{d}$. We have $\operatorname{dim} G_{2}\left(\right.$ Poly $\left._{d}\right)=2 d-2$. The maximal possible degree of the Wronskian of an element of $G_{2}\left(\right.$ Poly $\left._{d}\right)$ is $2 d-2$. Polynomials of degree $\leq 2 d-2$, considered up to a nonzero factor, form a $(2 d-2)$-dimensional complex projective space. Thus the mapping of $V \in G_{2}\left(\right.$ Poly $\left._{d}\right)$ into the Wronskian is a well-defined map of smooth complex algebraic varieties of the same dimension, $G_{2}\left(\right.$ Poly $\left._{d}\right) \rightarrow \mathbb{C P}^{2 d-2}$, called the Wronski map. The preimage of any monic polynomial consists of a finite number of planes. On Wronski maps see $\mathbb{E G}$.

2.3. Schubert calculus (Ch. 6 of $[\mathrm{GH}])$. Let $G_{2}(d+1)$ be the Grassmannian variety of two-dimensional subspaces $V \subset \mathbb{C}^{d+1}$. A chosen basis $e_{1}, \ldots, e_{d+1}$ of $\mathbb{C}^{d+1}$ defines the flag of linear subspaces

$$
E_{\bullet}: E_{1} \subset E_{2} \subset \ldots \subset E_{d} \subset E_{d+1}=\mathbb{C}^{d+1},
$$

where $E_{i}=\operatorname{Span}\left\{e_{1}, \ldots, e_{i}\right\}, \operatorname{dim} E_{i}=i$. For any integers $a_{1}$ and $a_{2}$ such that $0 \leq a_{2} \leq$ $a_{1} \leq d-1$, the Schubert variety $\Omega_{a_{1}, a_{2}}\left(E_{\bullet}\right) \subset G_{2}(d+1)$ is defined as follows, $\Omega_{a_{1}, a_{2}}=\Omega_{a_{1}, a_{2}}\left(E_{\bullet}\right)=\left\{V \in G_{2}(d+1) \mid \operatorname{dim}\left(V \cap E_{d-a_{1}}\right) \geq 1, \operatorname{dim}\left(V \cap E_{d+1-a_{2}}\right) \geq 2\right\}$. The variety $\Omega_{a_{1}, a_{2}}=\Omega_{a_{1}, a_{2}}\left(E_{\bullet}\right)$ is an irreducible closed subvariety of $G_{2}(d+1)$ of the complex codimension $a_{1}+a_{2}$.

The homology classes $\left[\Omega_{a_{1}, a_{2}}\right]$ of Schubert varieties $\Omega_{a_{1}, a_{2}}$ are independent of the choice of flag, and form a basis for the integral homology of $G_{2}(d+1)$. Define $\sigma_{a_{1}, a_{2}}$ to be the cohomology class in $H^{2\left(a_{1}+a_{2}\right)}\left(G_{2}(d+1)\right)$ whose cap product with the fundamental class of $G_{2}(d+1)$ is the homology class $\left[\Omega_{a_{1}, a_{2}}\right]$. The classes $\sigma_{a_{1}, a_{2}}$ are called Schubert classes. They give a basis over $\mathbb{Z}$ for the cohomology ring of the Grassmannian. The product or intersection of any two Schubert classes $\sigma_{a_{1}, a_{2}}$ and $\sigma_{b_{1}, b_{2}}$ has the form

$$
\sigma_{a_{1}, a_{2}} \cdot \sigma_{b_{1}, b_{2}}=\sum_{c_{1}+c_{2}=a_{1}+a_{2}+b_{1}+b_{2}} C\left(a_{1}, a_{2} ; b_{1}, b_{2} ; c_{1}, c_{2}\right) \sigma_{c_{1}, c_{2}},
$$

where $C\left(a_{1}, a_{2} ; b_{1}, b_{2} ; c_{1}, c_{2}\right)$ are nonnegative integers called the Littlewood-Richardson coefficients.

If the sum of the codimensions of classes equals $\operatorname{dim} G_{2}(d+1)=2 d-2$, then their intersection is an integer (identifying the generator of the top cohomology group $\sigma_{d-1, d-1} \in H^{4 d-4}\left(G_{2}(d+1)\right)$ with $\left.1 \in \mathbb{Z}\right)$ called the intersection number. 
When $\left(a_{1}, a_{2}\right)=(q, 0), 0 \leq q \leq d-1$, the Schubert varieties $\Omega_{q, 0}$ are called special and the corresponding cohomology classes $\sigma_{q}=\sigma_{q, 0}$ are called special Schubert classes.

2.4. Planes with a given Wronskian. In order to apply the Schubert calculus to our problem, we need the following result.

Proposition 2. Let $V \in G_{2}\left(\right.$ Poly $\left._{d}\right)$ be a generic plane of degree $d$ and order $k$ with the Wronskian $W(x)$. Then

- almost all polynomials of degree $d$ in $V$ do not have multiple roots;

- the polynomials of degree $k$ in $V$ are all proportional;

- if there exists a polynomial $p(x) \in V$ which has a root of multiplicity 1 at 0 , then $W(0) \neq 0$ and

$$
\frac{W^{\prime}(0)}{W(0)}=\frac{p^{\prime \prime}(0)}{p^{\prime}(0)} \text {. }
$$

- if 0 is a root of multiplicity $m \geq 1$ of $W(0)$, then there exists a polynomial in $V$ which has a root of multiplicity $m+1$ at 0 .

Proof: The first two statements are evident.

In order to prove the third statement, take a linearly independent polynomial $q(x) \in$ $V$. We have $q(0) \neq 0$ and $W[p, q](x)=p^{\prime}(x) q(x)-p(x) q^{\prime}(x)=c W(x)$ for some constant $c \neq 0$. The substitution of $x=0$ gives $c W(0)=p^{\prime}(0) q(0) \neq 0$. Moreover $c W^{\prime}(x)=$ $p^{\prime \prime}(x) q(x)-p(x) q^{\prime \prime}(x)$, and the substitution of $x=0$ into

$$
\frac{W^{\prime}(x)}{W(x)}=\frac{p^{\prime \prime}(x) q(x)-p(x) q^{\prime \prime}(x)}{p^{\prime}(x) q(x)-p(x) q^{\prime}(x)}
$$

finishes the proof of the third statement.

In order to prove the last statement, take a basis of $V,\{f(x), g(x)\}$ such that $\operatorname{deg} f<$ $\operatorname{deg} g$. We have $W(x)=x^{m} \tilde{W}(x)$, where $\tilde{W}(0) \neq 0$. There are two possibilities: $f(0)=0$ or $f(0) \neq 0$.

1) If $f(0)=0$, then $g(0) \neq 0$ and $f(x)$ has the form $f(x)=x^{k} \tilde{f}(x)$, where $\tilde{f}(0) \neq 0$, for some $k>1$. The Wronskian of $f$ and $g$, which is proportional to $W(x)$, has the form $x^{k-1} F(x)$, where $F(0)=k \tilde{f}(0) g(0) \neq 0$. Therefore $k=m+1$.

2) If $f(0) \neq 0$, then

$$
g_{0}(x)=g(x)-\frac{g(0)}{f(0)} f(x) \in V
$$

satisfies $g_{0}(0)=0$, and polynomials $f(x), g_{0}(x)$ form a basis of $V$. The rest of the proof is the same as in case 1$)$. 
2.5. The proof of (3). Now we are able to estimate from above the number of generic planes of degree $d$ with the Wronskian

$$
W(x)=W(x ; \mathbf{z}, \mathbf{m})=\left(x-z_{1}\right)^{m_{1}} \ldots\left(x-z_{n}\right)^{m_{n}}, \quad \operatorname{deg} W=M .
$$

For any fixed $z \in \mathbb{C}$, define the flag $\mathcal{F}_{\bullet}(z)$ in Poly $_{d}$,

$$
\mathcal{F}_{\bullet}(z): \mathcal{F}_{0}(z) \subset \mathcal{F}_{1}(z) \subset \ldots \mathcal{F}_{d}(z)=\text { Poly }_{d}, \quad \operatorname{dim} \mathcal{F}_{i}(z)=i+1,
$$

where $\mathcal{F}_{i}(z)$ consists of the polynomials of the form

$$
a_{i}(x-z)^{d-i}+\cdots+a_{0}(x-z)^{d} .
$$

Define $\mathcal{F}_{\bullet}(\infty)$ as the flag with $\mathcal{F}_{i}(\infty)=$ Poly $_{i}, 0 \leq i \leq d$.

Let $V$ be a generic plane of degree $d$ with the Wronskian given by (7) and (2).

According to Proposition 2, for any $z_{j}$ there exists a basis of $V$ such that one of the polynomials does not vanish at $z_{j}$ and the other has a root at $z_{j}$ of multiplicity $m_{j}+1$. This exactly means that $V \in \Omega_{m_{j}, 0}\left(\mathcal{F}_{z_{j}}\right)$.

The Wronskian of $V$ has degree $M$, therefore $V$ should contain a polynomial of degree $M+1-d$. Thus $V$ has a basis of polynomials of degrees $d$ and $M+1-d$, i.e. $V \in$ $\Omega_{2 d-2-M, 0}\left(\mathcal{F}_{\infty}\right)$.

Thus we conclude that

$$
V \in \Omega_{m_{1}, 0}\left(\mathcal{F}_{\bullet}\left(z_{1}\right)\right) \cap \Omega_{m_{2}, 0}\left(\mathcal{F}_{\bullet}\left(z_{2}\right)\right) \cap \cdots \cap \Omega_{m_{n}, 0}\left(\mathcal{F}_{\bullet}\left(z_{n}\right)\right) \cap \Omega_{2 d-2-M, 0}\left(\mathcal{F}_{\bullet}(\infty)\right) .
$$

The dimension of $G_{2}\left(\right.$ Poly $\left._{d}\right)$ is the sum of the codimensions of these Schubert varieties, therefore the intersection consists of a finite number of planes. This number does not exceed $\sigma_{m_{1}} \cdot \ldots \cdot \sigma_{m_{n}} \cdot \sigma_{2 d-2-M}$.

\section{The MASTER FUnCTION}

3.1. Fuchsian differential equations with only polynomial solutions. Consider a second order Fuchsian differential equation with regular singular points at $z_{1}, \ldots, z_{n}$, $n \geq 2$, and at infinity. If the exponents at $z_{j}$ are 0 and $m_{j}+1,1 \leq j \leq n$, then this equation has the form

$$
\begin{gathered}
F(x) u^{\prime \prime}(x)+G(x) u^{\prime}(x)+H(x) u(x)=0, \\
F(x)=\prod_{j=1}^{n}\left(x-z_{j}\right), \quad \frac{G(x)}{F(x)}=\sum_{j=1}^{n} \frac{-m_{j}}{x-z_{j}},
\end{gathered}
$$

where $H(x)$ is a polynomial of degree not greater than $n-2$. On Fuchsian equations see Ch. 6 of $[\mathrm{R}]$. As before, we write $M=m_{1}+\cdots+m_{n}$.

Proposition 3. Let $m_{1}, \ldots, m_{n}, n \geq 2$, and $d$ be positive integers such that $d>M+$ $1-d \geq 0$. There is a one-to-one correspondence between the generic planes of degree $d$ with the Wronskian (7) and the equations (8) with only polynomial solutions and the degree d generic solutions. 
Proof: The Wronskian of the equation (8) is exactly the polynomial $W(x)$ given by (匹). If this equation has only polynomial solutions and if the generic solution has degree $d$, then the solution space is a plane in $G_{2}\left(\right.$ Poly $\left._{d}\right)$ of degree $d$. If $g(x)$ and $f(x)$ form a basis in the solution space, then the Wronskian of $g$ and $f$ is proportional to $W(x)$. Therefore $g$ and $f$ may have a common root at points $z_{j}$ only. But the generic solution

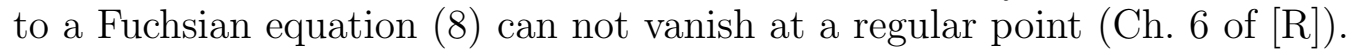

In order to prove the opposite statement, consider a basis $\{g(x), f(x)\}$ of a generic plane $V$ of degree $d$ with the Wronskian (7). We can assume $f(x)=\left(x-t_{1}\right) \cdots\left(x-t_{d}\right)$ where the roots $t_{1}, \ldots, t_{d}$ satisfy

$$
t_{i} \neq t_{l}, t_{i} \neq z_{j}, 1 \leq i, l \leq d, 1 \leq j \leq n,
$$

according to Proposition 2. The plane $V$ is the solution space of the following second order linear differential equation with respect to unknown function $u(x)$,

$$
\left|\begin{array}{ccc}
u(x) & f(x) & g(x) \\
u^{\prime}(x) & f^{\prime}(x) & g^{\prime}(x) \\
u^{\prime \prime}(x) & f^{\prime \prime}(x) & g^{\prime \prime}(x)
\end{array}\right|=0 .
$$

The Wronskian of the polynomials $f(x)$ and $g(x)$ is proportional to $W(x)$ given by (7), therefore this equation can be re-written in the form

$$
W(x) u^{\prime \prime}(x)-W^{\prime}(x) u^{\prime}(x)+h(x) u(x)=0,
$$

where $h(x)$ is a polynomial proportional to the Wronskian of $f^{\prime}(x)$ and $g^{\prime}(x)$. One can easily check that

$$
\frac{W^{\prime}(x)}{W(x)}=\sum_{j=1}^{n} \frac{m_{j}}{x-z_{j}}
$$

Moreover, we have

$$
h(x)=\frac{-W(x) f^{\prime \prime}(x)+W^{\prime}(x) f^{\prime}(x)}{f(x)},
$$

as $f(x)$ is clearly a solution to this equation. Hence if $z_{j}$ is a root of $W(x)$ of multiplicity $m_{j}>1$, then all coefficients of the equation have $\left(x-z_{j}\right)^{m_{j}-1}$ as a common factor, and the equation can be reduced to the required form (8).

Corollary 1. Any plane in $G_{2}$ (Poly) with a given Wronskian is uniquely determined by any of its polynomial.

The following statement easily follows from the theory of Fuchsian equations (Ch. 6 of $[\mathbb{R}]$ ).

Proposition 4. (Cf. Sec. 3.1 of [SV]) If the equation (8) has a polynomial solution without multiple roots, then all solutions to this equation are polynomials. 
3.2. The proof of the left-hand inequality of (6). Denote

$$
Z=\left\{\mathbf{z}=\left(z_{1}, \ldots, z_{n}\right) \in \mathbb{C}^{n} \mid z_{i} \neq z_{j}, 1 \leq i<j \leq n\right\} .
$$

Let $\mathbf{z} \in Z$ be fixed and $W(x)=W(x ; \mathbf{z}, \mathbf{m})$ be given by (17). Define a symmetric function in $k$ complex variables $\mathbf{t}=\left(t_{1}, \ldots, t_{k}\right)$,

$$
\Phi(\mathbf{t})=\Phi_{k, n}(\mathbf{t} ; \mathbf{z}, \mathbf{m})=\frac{\prod_{j<l}\left(t_{j}-t_{l}\right)^{2}}{\prod_{i=1}^{k} W\left(t_{i}\right)},
$$

in the domain

$$
T=T_{k, n}(\mathbf{t} ; \mathbf{z})=\left\{\mathbf{t} \in \mathbb{C}^{k} \mid t_{i} \neq t_{l}, t_{i} \neq z_{j}, 1 \leq i, l \leq k, 1 \leq j \leq n\right\} .
$$

The function $\Phi(\mathbf{t})$ is called the master function.

$A$ critical point of the function $\Phi(\mathbf{t})$ is a point $\mathbf{t}^{0} \in T$ such that

$$
\frac{\partial \Phi}{\partial t_{i}}\left(\mathbf{t}^{0}\right)=0, \quad i=1, \ldots, k .
$$

For any plane in $G_{2}$ (Poly) with the Wronskian of degree $M$, the sum of the order and of the degree is $M+1$. Under the assumptions (2), we have $0<M+1-d<d$.

Heine and Stieltjes, in their studies of second order linear differential equations with polynomial coefficients and a polynomial solution of a prescribed degree, arrived at the result which can be formulated as follows.

Proposition 5. (Cf. [SZ], Ch. 6.8) Let $\mathbf{z} \in Z$ be fixed, $n, d, m_{1}, \ldots, m_{n}$ be positive integers satisfying (2), $M=m_{1}+\cdots+m_{n}, k=M+1-d$.

If $\mathbf{t}^{0}$ is a critical point of the master function (10), then $f(x)=\left(x-t_{1}^{0}\right) \ldots\left(x-t_{k}^{0}\right)$ is a solution to the equation (8).

Conversely, if $\mathbf{t}^{0} \in T$ and if $f(x)=\left(x-t_{1}^{0}\right) \ldots\left(x-t_{k}^{0}\right)$ is a solution to the equation (8), then $t^{0}$ is a critical point of the master function (10).

The symmetric group $S^{k}$ acts on $T$ permuting $t_{1}, \ldots, t_{k}$, and the action preserves the critical set of the master function. Say that a generic plane $V \in G_{2}$ (Poly) of order $k>0$ is nondegenerate, if the polynomials of degree $k$ in $V$ do not have multiple roots. The nondegenerate planes correspond to the classes of rational functions which can be represented by the ratio of polynomials without multiple roots. Propositions 4 and 5 imply the following result.

Corollary 2. Let $\mathbf{z} \in Z$ be fixed, $W(x)=W(x ; \mathbf{z}, \mathbf{m})$ be given by (7)), (2), $k=M+1-d$, and $\Phi(\mathbf{t})=\Phi_{k, n}(\mathbf{t} ; \mathbf{z}, \mathbf{m})$ be given by (10). There is a one-to-one correspondence between the orbits of critical points of the master function $\Phi(\mathbf{t})$ and the nondegenerate planes of degree $d$ with the Wronskian $W(x)$.

Any nondegenerate plane is generic, and in order to get the left-hand inequality of (6) it remains to use Proposition 11.

\section{Remarks:}


- For $k=1$, the master function is $\Phi_{n, 1}(t ; \mathbf{z}, \mathbf{m})=W^{-1}(t)$, and the critical points are exactly those of the critical points of $W(x)$ which have a non-zero critical value.

- According to Proposition 2, if $f(x)=\left(x-t_{1}^{0}\right) \ldots\left(x-t_{k}^{0}\right)$ is a polynomial without multiple roots in a nondegenerate plane of degree $d$, of order $k=M+1-d$ and with the Wronskian $W(x)$, then $\mathbf{t}^{0}$ is a solution to the system

$$
\frac{W^{\prime}\left(t_{i}\right)}{W\left(t_{i}\right)}=\frac{f^{\prime \prime}\left(t_{i}\right)}{f^{\prime}\left(t_{i}\right)}, \quad i=1, \ldots, k .
$$

This is exactly the critical point system of the master function.

- After the first version of this paper was posted on arXiv, the author received from A. Eremenko (unpublished) notes where the connection between classes of rational functions and Fuchsian equations with trivial monodromy, in the case $n=2 d-2$ and $m_{1}=\cdots=m_{n}=1$, has been established.

\section{A lower Bound: ASYmptotic solutions to the $s l_{2}$ KZ EQUATion}

4.1. The $s l_{2} \mathbf{K Z}$ equation $\left.(\mathbb{R Z}]\right)$. Consider the Lie algebra $s l_{2}=s l_{2}(\mathbb{C})$ with the standard basis $e, f, h$ such that $[e, f]=h,[h, e]=2 e,[h, f]=-2 f$.

For positive integers $m_{1}, \ldots, m_{n}, n \geq 2$, let $L_{m_{j}}$ be the irreducible $s l_{2}$-module with highest weight $m_{j}$, and $L=L_{m_{1}} \otimes \cdots \otimes L_{m_{n}}$ the tensor product.

For the Casimir element,

$$
\Omega=e \otimes f+f \otimes e+\frac{1}{2} h \otimes h \in s l_{2} \otimes s l_{2},
$$

and for $1 \leq i<j \leq n$, let $\Omega_{i j}: L \rightarrow L$ be the operator which acts as $\Omega$ on $i$-th and $j$-th factors of $L$ and as the identity on all others. For $\mathbf{z} \in Z$, see (9), define operators $H_{1}(\mathbf{z}), \ldots, H_{n}(\mathbf{z})$ on $L$ as follows,

$$
H_{i}(\mathbf{z})=\sum_{j \neq i} \frac{\Omega_{i j}}{z_{i}-z_{j}}, \quad i=1, \ldots, n .
$$

The Knizhnik-Zamolodchikov (KZ) equation on a function $u: Z \rightarrow L$ is the system of partial differential equations

$$
\kappa \frac{\partial u}{\partial z_{i}}=H_{i}(\mathbf{z}) u(\mathbf{z}), \quad i=1, \ldots, n,
$$

where $\kappa$ is a parameter. This equation appeared first in Wess-Zumino models of conformal field theory, $\mathrm{KZ}$.

4.2. Subspaces of singular vectors. Let $k$ be a nonnegative integer, $k \leq M / 2$ (as before, $\left.M=m_{1}+\cdots+m_{n}\right)$. Denote $\operatorname{Sing}_{k} L$ the subspace of singular vectors of weight $M-2 k$ in $L$,

$$
\operatorname{Sing}_{k} L=\{w \in L \mid e w=0, h w=(M-2 k) w\} .
$$


4.3. The hypergeometric solutions to the $\mathrm{KZ}$ equation. The KZ equation preserves $\operatorname{Sing}_{k} L$ for any $k$. In [ScV], the hypergeometric solutions to the KZ equation with values in $\operatorname{Sing}_{k} L$ were constructed. Consider the function

$$
\Psi(\mathbf{t}, \mathbf{z})=\prod_{1 \leq i<j \leq n}\left(z_{i}-z_{j}\right)^{m_{i} m_{j} / 2} \prod_{i=1}^{k} \prod_{l=1}^{n}\left(t_{i}-z_{l}\right)^{-m_{l}} \prod_{1 \leq i<j \leq k}\left(t_{i}-t_{j}\right)^{2}
$$

defined on

$$
\mathcal{C}=\mathcal{C}_{k, n}(\mathbf{t}, \mathbf{z})=\left\{\mathbf{t} \in \mathbb{C}^{k}, \mathbf{z} \in \mathbb{C}^{n} \mid t_{i} \neq t_{l}, z_{j} \neq z_{p}, t_{i} \neq z_{j}, 1 \leq i, l \leq k, 1 \leq j, p \leq n\right\} .
$$

For the natural projection $\mathcal{C}_{k, n}(\mathbf{t}, \mathbf{z}) \rightarrow Z,(\mathbf{t}, \mathbf{z}) \mapsto \mathbf{z}$, the preimage of $\mathbf{z}$ is $T_{k, n}(\mathbf{t} ; \mathbf{z})$, see (9), (11). In Sec. 7 of [ScV], the solutions to the KZ equation (13) with values in $\operatorname{Sing}_{k} L$ were constructed. They have the form

$$
u(\mathbf{z})^{\gamma(\mathbf{z})}=\int_{\gamma(\mathbf{z})} \Psi^{1 / \kappa}(\mathbf{t}, \mathbf{z}) R(\mathbf{t}, \mathbf{z}) d t_{1} \wedge \cdots \wedge d t_{k},
$$

where $\gamma(\mathbf{z})$ is a $k$-cycle in $T=T_{k, n}(\mathbf{t} ; \mathbf{z})$ continuously depending on $\mathbf{z}$, and $R(\mathbf{t}, \mathbf{z})$ is a certain rational function on $\mathcal{C}$ with values in $\operatorname{Sing}_{k} L$.

\subsection{The proof of the right-hand inequality of (6). The series}

$$
\phi(\mathbf{z})=e^{I(\mathbf{z}) / \kappa}\left(\phi_{0}(\mathbf{z})+\kappa \phi_{1}(\mathbf{z})+\kappa^{2} \phi_{2}(\mathbf{z})+\ldots\right),
$$

where $I$ and $\phi_{i}$ are functions on $Z$, provides an asymptotic solution to the equation (13) if the substitution of $\phi$ into (13) gives 0 in the expansion into a formal power series in $\kappa$. If $\phi(\mathbf{z})$ is an asymptotic solution, then the substitution into (13) shows that $\phi_{0}(\mathbf{z}) \in \operatorname{Sing}_{k} L$ is an eigenvector of $H_{j}(\mathbf{z})$ for any $1 \leq j \leq n$.

Asymptotic solutions can be produced by taking the limit as $\kappa \rightarrow 0$ of $u(\mathbf{z})^{\gamma(\mathbf{z})}$ given by (15). According to the steepest descend method, the leading terms $\phi_{0}(\mathbf{z})$ are determined by critical points of the function $\Psi^{1 / \kappa}(\mathbf{t}, \mathbf{z})$ with respect to $\mathbf{t}$. In order to study the critical points, one can clearly replace $\Psi^{1 / \kappa}(\mathbf{t}, \mathbf{z})$ with the function

$$
\Phi(\mathbf{t})=\prod_{i=1}^{k} \prod_{l=1}^{n}\left(t_{i}-z_{l}\right)^{-m_{l}} \prod_{1 \leq i<j \leq k}\left(t_{i}-t_{j}\right)^{2}
$$

defined on $T_{k, n}(\mathbf{t} ; \mathbf{z})$ given by (11). This is exactly the master function (10).

Proposition 6. (Theorem 9.9 of [RV], see also Theorem 8 of [SV])

- Any orbit of nondegenerate critical point $\mathbf{t}^{0}(\mathbf{z})$ of the master function $\Phi(\mathbf{t})$ defines a common eigenvector $w\left(\mathbf{t}^{0}, \mathbf{z}\right)$ of operators $H_{1}(\mathbf{z}), \ldots, H_{n}(\mathbf{z})$.

- For generic $\mathbf{z} \in Z$, the eigenvectors $w\left(\mathbf{t}^{0}, \mathbf{z}\right)$ generate the space $\operatorname{Sing}_{k} L$.

Thus the number of orbits of critical points of the master function (10) is at least $\operatorname{dim} \operatorname{Sing}_{k} L$. 


\section{Comments}

5.1. A Hurwitz problem. The question about the number of rational maps with prescribed singularities is a classical one.

In 1891, A. Hurwitz published a formula giving the number of some special factorizations of a permutation into transpositions, $[\mathrm{H}]$. This formula immediately yields the number of topologically non-equivalent rational functions on $\mathbb{C}^{1}$ with fixed orders of poles and fixed critical values, in the case when all critical points are nondegenerate and all critical values are different. The topological equivalence of two functions means that one turns into the other by a linear-fractional transformation of the domain.

Concerning the proof of the formula, there was a kind of discussion whether A. Hurwitz had solved it completely, or his proof contains a gap. Since then, many papers on the subject were appeared. Let us mention the paper of I.P. Goulden and D.M. Jakson GJ] where the formula was rediscovered in its full, cf. [M].

The question posed in the Introduction differs from the Hurwitz's one. Our setting follows that of $[\mathrm{G}]$, EG], cf. [EH]. Firstly, not critical values but critical points are fixed. Secondly, a degree is fixed as well. Thirdly, the equivalence relation is defined by linear-fractional transformations of the target, not the domain.

5.2. Multiplicities of $s l_{2}$-representations. Formula (5) gives the multiplicity of $L_{M-2 k}$ in the decomposition of the tensor product $L_{m_{1}} \otimes \ldots \otimes L_{m_{n}}$ into the direct sum of irreducible $s l_{2}$-modules. The proof of the formula given in [SV] is short. It involves in the Inclusion-Exclusion principle and elementary $s l_{2}$-representation theory. However we were unable to find this formula in the literature as on representation theory as on the Schubert calculus.

5.3. Degrees of Wronski maps and Catalan numbers. The Wronski map sends the big cell of $G_{2}\left(\right.$ Poly $\left._{d}\right)$ (the planes of degree $d$ and order $d-1$ ) to the big cell of the projective space (the monic polynomials of degree exactly $2 d-2$ ). Consider the restriction of the Wronski map to the big cell. The cardinality of a generic preimage is called the degree of the Wronski map, EG.

The main result of $\mathbb{G}$ says that over $\mathbb{C}$ the degree of the Wronski map equals the Catalan number $C_{d}=\frac{1}{d}\left(\begin{array}{c}2 d-2 \\ d-1\end{array}\right)$. In our notation, $C_{d}=\sharp(d, 2 d-2 ; \mathbf{1} ; \mathbf{z})$, where $\mathbf{1}=$ $(1, \ldots, 1)$. The Catalan number gives also an upper bound for the number of the classes of rational maps of the type $(d, n ; \mathbf{m} ; \mathbf{z})$ with $m_{1}+\cdots+m_{n} \leq 2 d-2([\mathbf{G})$. In [EG], upper and lower estimates for the number of classes of real rational maps of degree $d$ in terms of the Catalan numbers were established.

The Catalan numbers appear as the answer to many combinatorial problems (see for example [St], Ex. 6.19 and 6.25). We get that the $(k+1)$-th Catalan number is the multiplicity of $L_{0}$ in $L_{1}^{\otimes 2 k}$. This interpretation leads to the following conclusion. 
Proposition 7. The generating function for the multiplicity $M_{k}$ of the trivial sl $l_{2}$ representation $L_{0}$ in $L_{1}^{\otimes k}$ is

$$
M(\tau)=\frac{1-2 \tau^{2}-\sqrt{1-4 \tau^{2}}}{2 \tau^{2}}=\sum_{k=1}^{\infty} M_{k} \tau^{k}, \quad \tau \rightarrow 0 .
$$

Indeed, the generating function for the Catalan numbers $C_{k}$ is as follows, [St],

$$
C(\tau)=\frac{1-\sqrt{1-4 \tau}}{2}=\sum_{k=1}^{\infty} C_{k} \tau^{k}, \quad \tau \rightarrow 0,
$$

and the multiplicity of $L_{0}$ in $L_{1}^{\otimes 2 k-1}$ is obviously zero. Therefore we have

$$
M(\tau)=\sum_{k=1}^{\infty} C_{k+1} \tau^{2 k}=\frac{C\left(\tau^{2}\right)}{\tau^{2}}-1
$$

This gives the statement.

\subsection{The Schubert calculus and Fuchsian equations with only polynomial so- lutions. One of the main results of [SV] is as follows.}

Proposition 8. (Theorem 3 of [SV]) Let $m_{1}, \ldots, m_{n}$, and $k$ be nonnegative integers, $n \geq 2, M=m_{1}+\cdots+m_{n}$ and $k \leq M / 2$. Then for generic $\mathbf{z} \in Z$ there exist exactly

$$
\sum_{q=0}^{n}(-1)^{q} \sum_{1 \leq i_{1}<\cdots<i_{q} \leq n}\left(\begin{array}{c}
k+n-2-m_{i_{1}}-\cdots-m_{i_{q}}-q \\
n-2
\end{array}\right)
$$

polynomials $H(x)$ of degree not greater than $n-2$ such that all solutions to the equation (8) are polynomials and the degree of the generic solution equals $M+1-k$.

The proof given in $\|S \nabla\|$ is rather complicated. Using of the Schubert calculus leads to another, "less technical", proof, in view of Propositions 1, 3.

The theory of Fuchsian differential equations of order $p$ with only polynomial solutions is closely related to the Schubert calculus in the Grassmannian of $p$-dimensional subspaces of the vector space of complex polynomials. If the generic solution of such an equation has degree $d$, then the solution space $V$ belongs to $G_{p}\left(\right.$ Poly $\left._{d}\right)$. Let polynomials $P_{1}(x), \ldots, P_{p}(x)$ form a basis of $V \in G_{p}\left(\right.$ Poly $\left._{d}\right)$. Then the equation $E_{V}(u)=0$ with respect to unknown function $u$, where

$$
E_{V}(u)=\operatorname{det}\left(\begin{array}{cccc}
u(x) & P_{1}(x) & \ldots & P_{p}(x) \\
u^{\prime}(x) & P_{1}^{\prime}(x) & \ldots & P_{p}^{\prime}(x) \\
\ldots & \ldots & \ldots & \ldots \\
u^{(p)}(x) & P_{1}^{(p}(x) & \ldots & P_{p}^{(p)}(x)
\end{array}\right),
$$

is Fuchsian and its solution space is $V$. The Wronskian $W_{V}(x)$ of $V$ is defined as a monic polynomial which is proportional to the Wronskian of some (and hence, any) basis of $V$, cf. EG]. The Wronskian of the equation $E_{V}(u)=0$ is proportional to $W_{V}(x)$, and the 
regular singular points of the equation are the roots of $W_{V}(x)$ ("the root at infinity" has multiplicity $\left.p(d+1-p)-\operatorname{deg} W_{V}\right)$. For any $z \in \mathbb{C} \cup \infty$, the set of exponents at $z$ defines a Schubert variety $\Omega_{w(z)}\left(\mathcal{F}_{\bullet}(z)\right)$ containing $V(\mathrm{CH} .6$ of GH], cf. Sec. 2.5). In particular, $z$ is an ordinary point of the equation if and only if $w(z)=(0, \ldots, 0)$. The intersection of the Schubert varieties corresponding to all regular singular points consists of a finite number of elements. Thus the number of Fuchsian differential equations of order $p$ with only polynomial solutions, having given regular singular points, given exponents at these points and the degree $d$ generic solution, is bounded from above by the intersection number of the corresponding Schubert classes.

5.5. The Schubert calculus and the $s l_{p} \mathbf{K Z}$ equations. In our case, the theory of KZ equations helps to solve a problem from enumerative algebraic geometry. On the other hand, the Schubert calculus seems to be a useful tool in the theory of KZ equations. In particular, the function which appears in the hypergeometric solutions to the $s l_{p} \mathrm{KZ}$ equation plays the role of the master function for the Wronski map from the Grassmannian of $p$-dimensional planes, Ga], [S]. The Schubert calculus provides an upper bound for the number of orbits of critical points as the intersection number of appropriate Schubert classes. For the tensor product of symmetric powers of the standard $s l_{p}$-module, a lower bound can be obtained, combining the theory of Fuchsian equations and results on KZ equations, as the dimension of a relevant subspace of singular vectors, [S]. The coincidence of these upper and lower bounds implies non-trivial results both in the theory of $\mathrm{KZ}$ equations (the proof of the conjecture on Bethe vectors in Gaudin model) and in the enumerative algebraic geometry (the proof of the transversality claim for Schubert varieties). 


\section{REFERENCES}

[EG] A. Eremenko and A. Gabrielov, The Wronski map and Grassmannians of real codimension 2 subspaces, to appear in Computational Methods and Function Theory.

[EH] D. Eisenbud and J. Harris, Limit linear series: Basic theory, Invent. Math. 85 (1986), 337 371.

[F] W. Fulton, "Young Tableaux", Cambridge University Press, 1997.

[Ga] A. Gabrielov, private communication.

[G] L. Goldberg, Catalan Numbers and Branched Coverings by the Riemann Sphere, Advances in Mathematics 85 (1991), 129-144.

[GH] Ph. Griffiths and J. Harris, Principles of Algebraic Geometry, Wiley, New York, 1978.

[GJ] I.P. Goulden and D.M. Jakson, Transitive factorizations into transpositions and holomorphic mappings on the sphere, Proc. Amer. Math. Soc. 125 (1997), 51-60.

$[\mathrm{H}] \quad$ A. Hurwitz, Über Riemann'sche Flächen mit gegebenen Verzweigungspunkten, Math. Ann. 39 (1891), 1-66.

[KZ] V. Knizhnik and A. Zamolodchikov, Current algebra and Wess-Zumino models in two dimensions, Nucl. Phys. B247 (1984), 83-103.

[M] A. Mednykh, Nonequivalent coverings of Riemann surfaces with a prescribed ramification type. (Russian) Sibirsk. Mat. Zh. 25 (1984), no. 4, 120-142.

[R] E. Rainville, Intermediate differential equations, The Macmillan Company, 1964.

[RV] N. Reshetikhin and A. Varchenko, Quasiclassical Asymptotics of Solutions to the KZ Equations. In: Geometry, Topology, and Physics for Raoul Bott, International Press, 1994, 293-322.

$[\mathrm{ScV}]$ V. Schechtman and A. Varchenko, Arrangements of hyperplanes and Lie algebra homology. Invent. Math. 106 (1991), 139 - 194.

[S] I. Scherbak, Asymptotic solutions to the $s l_{2} \mathrm{KZ}$ equation and the intersection of Schubert classes, preprint (2002), math.QA/0207218.

[SV] I. Scherbak and A. Varchenko, Critical points of functions, $s l_{2}$ representations, and Fuchsian differential equations with only univalued solutions, preprint (2001), math.QA/0112269.

[St] R. Stanley, Enumerative Combinatorics, Vol.2, Cambridge UP, Cambridge, 1999.

[Sz] G. Szego, Orthogonal polynomials, AMS, 1939. 